\title{
PER UNA STORIA DELLA STRUTTURA DELLA FRASE IN ITALIANO: IL FIORENTINO DEL CINQUECENTO
}

Nella storia dell'italiano la struttura della frase è cambiata più di una volta.

Con la struttura della frase cambiano anche le regole da cui dipendono: 1) la presenza e la posizione dei pronomi personali soggetto, 2) la posizione dei pronomi clitici obliqui adverbali, presenti in tutte le fasi dell'italiano come delle altre lingue romanze.

La prima fase è quella dell'italiano antico, cioè del fiorentino dalla prima documentazione, nel Duecento avanzato, al Quattrocento. Questa fase è illustrata, assieme a quella delle altre lingue romanze, in Vanelli, Renzi e Benincà 1985, Renzi 19904 e in corso di stampa, e più in dettaglio in Vanelli 1986 (per lo status teorico del tipo romanzo antico, v. Benincà 1983-84). Ne riprendo brevemente qui le linee essenziali, per passare poi al tema centrale di questo studio, la struttura del fiorentino del Cinquecento. Accennerò poi al tema della separazione dell'italiano letterario dal fiorentino e agli svolgimenti successivi divergenti dell'italiano e del fiorentino.

1. In italiano antico l'ordine degli elementi fondamentali nella frase è diverso nella frase subordinata e nella principale.

Nella subordinata abbiamo SVO (Soggetto, Verbo, Oggetto), con soggetto pronominale obbligatorio. Questa struttura rappresenta l'ordine basico.

Nella principale abbiamo VSO che presenta l'avanzamento di V rispetto all'ordine della subordinata, oppure OVS, IVS, SVO e simili ottenuti partendo da SVO basico della subordinata tramite avanzamento di $\mathrm{V}$ e di un altro elemento (rispettivamente $\mathrm{O}, \mathrm{I}, \mathrm{S}$ ecc.). Nel complesso l'it.a. appare come una lingua "verb second". Il pronome personale soggetto appare obbligatoriamente quando il soggetto precede il verbo $\left(\mathrm{SV},{ }^{*}-\mathrm{V}\right)$, ma può mancare quando segue $\left(\mathrm{VS}, \mathrm{V}_{-}, \mathrm{OVS}, \mathrm{OV}_{-}\right.$, IVS, IV _ ecc.). Diversamente che in ital. mod., la presenza del pronome è dovuta a cause sintattiche, non pragmatiche (Vanelli 1986).

Una variante molto diffusa della precedente mostra gli ordini OVS, IVS, ecc. anche nella frase subordinata. Quando ciò avviene, il pronome personale soggetto può mancare anche nella subordinata nelle strutture OVS, IVS ecc. Se manca dove $\mathrm{V}$ non è preceduta da costituente, si può postulare che la struttura sia VS, documentata con soggetto lessicale. 
In tutte e due le varianti i pronomi soggetto sono liberi, non clitici. Infatti possono essere separati dal verbo e possono stare in isolamento (Renzi, Vanelli, Benincà 1985; Renzi 1983). Tuttavia nel corso del tempo si erano formate delle forme clitiche al verbo: gli per la 3.a sing. e pl. masch., la e le per la 3.a rispettivamente sing. e pl. femm. (v. Renzi 1983).

Quanto ai pronomi clitici obliqui, essi sono regolati dalla legge Tobler-Mussafia, per cui il pronome è enclitico al verbo finito se questo è in prima posizione, assoluta o dopo congiunzione (ess.: \# "andovi e provollo", Disc. cler. in Schiaffini 1926 , p. 75, 1), altrimenti proclitico ( $E$ cosi si mosse, ibid. 74, 26). Questa soluzione, secondo un'ipotesi, è il risultato della rianalisi della c.d. legge di Wackernagel, che in latino vigeva ancora solo per i pronomi, applicata alla nuova struttura della frase italiana (Renzi 1987).

2. Il fiorentino dell'uso vivo del Cinquecento presenta delle differenze notevoli rispetto alla prima fase. Praticamente è come se l'ordine diretto SVO della subordinata si estendesse alla principale. Ne consegue che il pronome personale soggetto, che era obbligatorio nella sola subordinata, essendo ora sempre preverbale, diventa obbligatorio in tutti i casi. Rimane la forma VSO per esprimere l'interrogativa, l'esclamativa e in pochi altri casi: qui l'espressione del pronome soggetto, benché frequente, non è del tutto obbligatoria, come non lo era nella fase antica.

Ma nel complesso, come nota giustamente Alan Freedman (1983, 175), "the frequent use and even super pleonastic use of the Subject Pronouns was characteristic of Florentine plebeian vernacular...".

La lingua di questa fase è documentata di alcune commedie fiorentine come quelle di Giovan Battista Gelli (1498-1563), che rappresentano in modo vivo la lingua parlata del tempo. ${ }^{1}$ Ulteriori assaggi potranno dire se altri autori possano essere altrettanto rappresentativi. Machiavelli appare meno avanzato del Gelli. In genere gli autori non fiorentini e anche fiorentini contemporanei hanno contaminato l'uso fiorentino contemporaneo con quello antico, presentato nel paragrafo precedente. Il quadro che ne viene è assai complesso e contradditorio (vedi Freedman 1983). Persistono gli ordini OVS, IVS e simili, mentre il numero dei pronomi personali espressi aumenta considerevolmente senza un apparente perché.

Nella lingua del Gelli invece, benché non manchino alcune incongruenze, il quadro è nel complesso chiaro, e possiamo credere ragionevolmente che rifletta l'uso vivo e coerente della lingua fiorentina contemporanea. E costituisce al tempo stesso, l'antecedente logico del fiorentino del Sette-Ottoçento al quale accenniamo al par. 3.

- Oltre alla Sporta, qui considerata, presentano più o meno lo stesso stato di lingua l'altra commedia del Gelli Lo errore, dello stesso Gelli. Anche la lingua dei Ragionamenti è degna di considerazione, ma negli sccritti più teorici il sistema si adegua in gran parte a quello della prosa alta, bembesca, contemporanea. 
Ecco una serie di casi tratti da Gelli, La sporta. ${ }^{2}$ Diamo, quando è possibile, due esempi di ordine SVO per persona: il primo in frase principale (reggente eventualmente una subordinata dallo stesso soggetto o con altro soggetto pronominale espresso). I numeri che seguono alle citazioni indicano l'atto e la scena):

1.a sing.:

Io t'arò forse a render ragione (I, 1)

2.a sing.:

E $t u$ mi pari una sciagurata $(I, 1)$

3.a sing. m.:

$E i$ dubita che com'ella intendesse che gli avesse dato moglie $(\mathrm{I}, 4)$

3.a sing. $f$.:

ell'ha ben ragione di aiutarci, ch'ella fu cagione ch'ell'avesse la pratica di questo giovane $(\mathrm{I}, 1)$

3.a esplet.:

egli è perché io ho di che $(I, 3)$

1.a pl.:

Noi sappiamo oramai ancor noi come va il mondo $(I, 3)$

2.a pl.:

E voi gridate che voi parete proprio un arrovellato $(\mathrm{I}, 1)$

3.a pl.m.:

se io tornassi a veder se e' sono dove io gli nascosi $(1,1)$

Ecco i ragionamenti lunghi, che voi avevate insieme, di quel che gli erano $(\mathrm{V}, 2)$.

3.a pl.f.:

Elle son vere d'avanzo (II, 5)

Oltre alle forme esemplificate sopra, abbiamo per la 1. pers. sing. $i$; per la 3.a sing. egli, e'; per la 3.a s. femm. la. pl.f. elleno.

I pronomi sono separabili dal verbo, come nei seguenti esempi, a eccezione di $l a$ e gli (come già in it.a. vedi sopra par. 1); come si vede, il pronome soggetto, oltre che obbligatorio, è libero, non clitico, come era in fiorentino antico e contrariamente a quanto avverrà nella fase successiva (avanti, par.4):

2 La Sporta è stata scritta nel 1543 e ristampata nel 1548 e nel 1550 . Citiamo dall'ed. Maestri basata sull'edizione del '50. Ai nostri fini, come ho potuto stabilire da un confronto con le altre edizioni, le differenze non sono rilevanti (ma vedi la nota seguente). 
Io, per me, non credo, compar mio, che l'abbia altro male... (I, 3)

E egli, per la sua miseria, non ci ha mai voluto mandare un medico $(1,1)$

Ecco dei casi di inversione, cioè di ordine VS, persona per persona. Il primo es. è sempre interrogativo, seguono in alcuni casi esempi di natura diversa. Ma adesso il nucleo dell'inversione diventa la struttura dell'interrogativa (con l'esclamativa, che ne ricalca la struttura). Gli altri casi, diversamente che nella prima fase, sono ormai marginali:

1.a sing.:

Non ti do io dua scudi al mese? (II, 1)

dich'io $(I, 1)$

sì che quivi non vo'io dirgli più nulla $(\mathrm{I}, 4)(\mathrm{Avv} \mathrm{V} \mathrm{S}$, secondo il modello della prima fase)

2.a sing.:

non odi $t u$ ? (II, 1)

3.a sing. m.:

Oh, fec'egli questo? $(\mathrm{V}, 2)$

Or non ci fosse egli chi stesse peggio di voi $(\mathrm{I}, 2)$ (esclamativa)

che a tutto sopperiv'egli (II, 1) (I V S, secondo il modello della prima fase)

3.a sing. f.:

che borbott'ella da sé la sciagurata? (I, 1)

Oh com'è ella viziata! (I, 1) (esclamativa con introduttore wh-)

1.a pl.:

Che? vorremmo noi star sempre in convinti? $(\mathrm{I}, 1)$

2.a pl.:

e dove volete voi ch'io vadia? $(I, 1)$

Oh! non ci fate voi mai far altro $(I, 1)$ (esclamativa $w h-$ )

3.a pl. m.:

... a che mi servon eglino? (II, 1)

3.a pl. f.:

Dove ne vann'ellen cosi insieme? (II, 3)

Vere son elleno (V, 2) (Agg V S, secondo il modello della prima fase)

Per quanto meno frequenti compaiono alcuni casi di interrogative senza pronome, previste dalla regola dell'it.a., e che in quel quadro non andrebbero considerate eccezioni, ma che contrastano con il sistema più avanzato. Gli esempi non sono nu- 
merosi. Eccone alcuni (il posto che avrebbe potuto essere occupato dal pronome soggetto è indicato con un trattino):

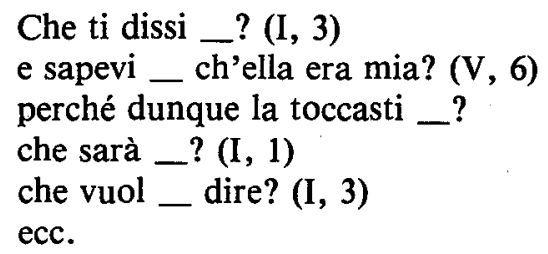

\section{In frase ottativa.}

almanco stian _ ferme (II, 4)

Questi casi possono essere ritenuti marginali, sia che siano degli arcaismi veri e propri, sia che rappresentino forme in via di eliminazione. La fase seguente presenterà dei pronomi soggetto enclitici obbligatori nella interrogativa. Bisogna pensare perciò che in questa fase, o eventualmente in una appena posteriore, il pronome posposto, ancora libero, fosse diventato obbligatorio.

Ci sono nel Gelli anche degli altri casi di mancanza di espressione del pronome. Bisogna distinguere tra i casi di eccezioni apparenti e quelli di eccezioni reali all'obbligatorietà del pronome. Cominciamo con le eccezioni apparenti: ${ }^{3}$

1) in coordinazioni di SV il pronome non è ripetuto. Questa non solo non è un'eccezione, ma al contrario è conforme alla regola di una lingua a pronome nonnullo, mentre la ripetizione obbligatoria del pronome è propria di una lingua in cui il pronome è in realtà da considerare un affísso, da cui la sua inamovibilità (vedi Rizzi 1985). Ess.:

Ei non esce quasi più fuora e tutto dì se ne va per casa di giù e di su; e or _ mi caccia di sala in cucina, or di cucina in sala; or _ non vuol... or _ non vuol... $(\mathrm{I}, 1)$.

3 Oltre ai casi considerati, ci sono anche alcune eccezioni apparenti al principio dell' obbligatorietà del soggetto che si eliminano per via filologica. Il caso seguente è un banale errore di stampa. Dove l'ediz. Maestri ha: "in modo che e' non par che e' sappia né quel che _ vuol ch'io faccia..."; l'edizione del 1548 aveva: "che e vuole" (dove $e=e^{\prime}$ ).

Così in I, 1 dove l'ediz. del ' 48 aveva: "e già son duoi mesi che a non ci ha mai comprato nulla", dove $a$ era un errore evidente per $e\left(=e^{\prime}\right)$. L'ediz. del ' 50 ha eliminato $a$ e cosi Maestri, provocando un'apparente assenza del pronome soggetto.

Altre eccezioni apparenti al principio dell'obbligatorietà del soggetto si possono eliminare in base a un'ipotesi filologica. Per es. in II, 1 dove è scritto "ché non le portava così tirate come vuoi far tu", si potrebbe interpretare "ch'e' non la portava"; e cosi in genere dove manca il pronome di 3.a persona dopo parola che esce in -e. Cosi forse anche in casi come II, 5: "che, come tu sai, gli sono stata vicina dietro di molt'anni", dove "sai, gli sono" si potrebbe leggere "sai $i$ " gli sono", dove "i"“ = "io". 
2) dove appare un ordine diverso da SVO, dove cioè il pronome soggetto seguirebbe il verbo (OV(S), IV(S) ecc.) il pronome può mancare, secondo la regola dell'italiano antico. Si tratta di casi minoritari che rappresentano un residuo letterario della lingua antica. Per es.:

Ben sai _ che io non sono il più ricco uomo del mondo $(I, 1)$

E per questo _ m'è entrato in sospetto, che... $(\mathrm{I}, 1)$

E, per disgrazia, stamane mi bisogna_ $(I, 1)$

ecc.

3) Dalla stessa ragione dipende il caso in cui il verbo è in posizione iniziale e il pronome manca. Quando il verbo è seguito da un pronome enclitico secondo la norma della cosiddetta legge Tobler-Mussafia è chiaro che abbiamo a che fare con la struttura antica:

Vedra'lo _ poi $(I, 1)$

e hacci — poi sempre mai aiutate $(I, 1)$

Nello stesso modo si possono interpretare anche delle forme senza pronome obliquo clitico;

Ho sentito _ non so chi bussare picchiare a casa mia (III, 2)

Anzi, crediam — appunto quello che s'ha da credere (III, 4)

- queste forme senza pronome si spiegano come forme dell'italiano antico, e secondo il principio dell'italiano antico che il pronome soggetto posposto al verbo può non apparire, come visto sopra. ${ }^{4}$

La scomparsa effettiva (nonostante la presenza di residui come questi) delle frasi a verbo iniziale di struttura VS ha portato con sé il tramonto della posizione enclitica dei pronomi. In questo senso non si può dire che a un certo momento la legge Tobler-Mussafia abbia cessato di funzionare, ma semplicemente è scomparsa la struttura che ne permetteva l'applicazione. (Naturalmente diversa strutturalmente è la forma a verbo iniziale dell'italiano moderno, che non è più VS con $\mathrm{S}$ non realizzato, cioè non "vedrai_“"), ma SV, cioè “_ vedrai“).

3. Mentre il fiorentino raggiungeva all'altezza del Gelli lo status di lingua a soggetto non-nullo, l'italiano letterario si dissociava dal fiorentino sotto l'influenza delle teorie del Bembo che imponeva il ritorno all'antico.

4 Come in italiano antico, che la forma sottostante delle frasi principali con soggetto non espresso sia VS e non SV non è dimostrabile, nel senso che non esiste nessuna prova che questa sia la forma di partenza e non quella col soggetto davanti al verbo (com'è per es. in ital. mod.). Ma l'ipotesi è coerente con il tipo di struttura postulato, e non ci sembra quindi illegittimo farne uso. 
3.1. Il fiorentino proseguiva il suo percorso carsico per affiorare alla fine del Settecento- inizio dell'Ottocento nelle commedie del Zannoni e del Nelli (Renzi 1983,1991 e in corso di stampa). I pronomi vi appaiono sempre obbligatori, ma non più liberi, ma clitici al verbo: proclitici nella frase dichiarativa (che avevano già cominciato a formarsi nella prima fase, v. sopra, par. 1), enclitici nella interrogativa $e$ nella esclamativa. Nel primo caso il pronome clitico può cooccorrere con il pronome libero, dando luogo alla cosiddetta reduplicazione, come nei seguenti ess. dallo Zannoni: E te tu l'hai com'e piedi dell'anatre". Interrogativa: "sa' tu, l'ha' tu sentita?" Questo sistema evolve in quello del fiorentino contemporaneo in cui i pronomi clitici passano ad affissi e come tali appaiono obbligatoriamente con soggetti nominali e pronominali, non mancano nemmeno nella coordinazione ecc. (Brandi 1981, Brandi e Cordin 1981, Renzi 1983, Bracco, Brandi e Cordin 1985, Rizzi 1985).

Questa evoluzione è del tutto parallela a quella del francese, fino all'ultima fase rappresentata dal français avancé (Renzi, in corso di stampa) e a quella dei dialetti italiani settentrionali in genere (Vanelli 1987).

Nessuna novità sul fronte dei clitici obliqui.

3.2. D'altro lato invece l'italiano letterario, benché si proponesse di imitare il sistema antico, evolveva anch'esso. L'ordine diretto SVO si impone sempre più, pure senza eliminare del tutto le forme con un costituente iniziale diverso dal soggetto. $\mathrm{Ma}$ in questo caso il posto del verbo non è più necessariamente il secondo. Così l'italiano moderno, diversamente da quello antico, non è più una lingua "verb second“.

Quanto ai pronomi soggetto, benché il Bembo si fosse limitato a censurare esplicitamente l'uso insorgente di lui soggetto (Prose della volgar lingua, libro III, XVI, cfr. Renzi 1983), il suo esempio è stato determinante per un ritorno al sistema antico in tutti i suoi aspetti, come è stato mostrato da Boström 1972 attraverso le autocorrezioni degli autori anche fiorentini del tempo.

Ma dalla fase cinquecentesca in poi il sistema italiano si contraddistingue per la fine dell'obbligatorietà del pronome provocata da ragioni sintattiche. In italiano moderno il pronome soggetto può essere obbligatorio solo per ragioni pragmatiche, non sintattiche.

Il sistema dell'italiano moderno è stato descritto con cura da Giampaolo Salvi, Paola Benincà, Lorenza Frison, Patrizia Cordin e Andrea Calabrese (vedi i capitoli I e XI della Grande Grammatica di Consultazione). Ma non è ancora stata descritta come risultato di uno sviluppo diacronico.

La descrizione di questo sviluppo è un compito che ci sta ancora davanti e che non sarà privo di implicazioni interessanti, come si vede già da adesso. 
Il sistema dell'italiano moderno è così il risultato di un'evoluzione "deviata" a partire non dal fiorentino vivo del Cinquecento, ma da quello antico restaurato. $\mathrm{Ma}$ si tratta pur sempre di un'evoluzione che fa sì che il sistema moderno sia una terza cosa rispetto a quello dell'italiano antico e a quello del fiorentino moderno. Tra il Cinquecento e l'età moderna, dunque, l'italiano si è mosso. Ciò contraddice l'idea che l'italiano letterario sia stato dal Cinquecento in poi "opera d'inchiostro", come diceva l'Ascoli, cioè una lingua artificiale scritta ma non parlata o quasi, come si è ripetuto troppe volte. Se le cose fossero state cosi ogni possibilità di sviluppo sarebbe stata bloccata. E' ora di rivedere quest'opinione, diventata ormai dogma.

A chi dice che l'italiano era una lingua morta, obietteremo: eppur si muoveva.

Notiamo infine che, a dispetto di tanti cambiamenti, in tutte le fasi viste, quella fiorentino-italiana, quelle solo fiorentine e solo italiane, accanto alle altre costruzioni c'è sempre quella con soggetto lessicale, anche definito, in posizione finale. Essa è documentata in fior.a., nel fiorentino del Cinquecento, in quello del Sette-Ottocento e in quello d'oggi. Gelli, senza pronome espletivo:

e se _ ci venisse la ventura... $(I, 2)$

e con pronome espletivo:

ché io so che come e' si scupre questa cosa, io ho a essere la male arrivata $(\mathrm{I}, 16)$

Come è noto, alla possibilità di apparizione del soggetto definito in posizione postverbale è stato attribuito valore diagnostico a partire da Perlmutter 1971. La possibilità di un soggetto postverbale definito sarebbe proprio delle sole lingue a soggetto nullo. Il fatto che questo carattere resti costante attraverso tanti cambiamenti di tipo pone un punto interrogativo sul valore diagnostico di questo test. Notiamo che dei dubbi in questione sono già stati sollevati, e che non è stato possibile trovare un accordo tra gli studiosi sul perché della correlazione ricordata. I fatti della storia dell'italiano pongono ora anche un punto interrogativo sul fondamento fattuale di questa correlazione.

\section{BIBLIOGRAFIA}

Testi

Opere di Giovan Battisti Gelli, a cura di D. Maestri, Torino, UTET, 1976.

Testi fiorentini del Dugento e dei primi del Trecento, a cura di A. Schiaffini, Firenze, Sansoni, 1926 (ristampa 1954). 
Studi

P. Benincà 1983-84, Un'ipotesi sulla sintassi delle lingue romanze medievali, in "Quaderni patavini di linguistica“, 3, pp. 3-19.

C. Bracco, L. Brandi, P. Cordin 1985, Sulla posizione soggetto in italiano e in alcuni dialetti dell'Italia centro-settentrionale, in Sintassi e morfologia della lingua italiana dell'uso, a cura di A. Franchi De Bellis e L.M. Savoia, SLI 24, pp. 185-209.

L. Brandi 1981, Sui clitici, in Studi di linguistica italiana per Giovanni Nencioni, Firenze, Tipografia Pappagallo, pp. 129-146.

L. Brandi, P. Cordin 1981, Dialetti e italiano: un confronto sul parametro del soggetto nullo, in "Rivista di grammatica generativa", 6, pp. 33-87.

A. Freedman 1983, "Vuoi tu murare?". The italian subject pronoun, in "Studi di grammatica italiana" XII, pp. 167-189.

Grande Grammatica italiana di consultazione, vol. I. La frase, I sintagmi nominale e preposizionale, a cura di L. Renzi, Bologna, Il Mulino, 1988, 1989.2

D. Perlmutter 1971, Deep and Surface Structure Constraints in Syntax, New York, Holt, Rinehart \& Winston.

Renzi, L. 1983, Fiorentino e Italiano: storia dei pronomi personali soggetto, in Italia linguistica: idee, storia, strutture, a cura di F. Albano Leoni e altri, Bologna, Il Mulino, pp. 223-239.

L. Renzi 1987, Essor, transformation et mort d'une loi: la loi de Wackernagel, in Mélanges offerts à M. Molho, vol. III: Linguistique, Les Cahiers de Fontenay, pp. 291-302.

L. Renzi $1990^{4}$, Nuova Introduzione alla filologia romanza, con la collaborazione di G. Salvi, Bologna, Il Mulino (1.a ed. 1985).

L. Renzi 1991, Per la storia del fiorentino: $i$ pronomi personali soggetto nelle commedie dello Zannoni, negli Atti del Congresso della SILFI a Siena.

L. Renzi in corso di stampa, I pronomi soggetto in due varietà substandard: fiorentino e français avancé, in corso di stampa in "Zeitschrift für romanische Philologie".

L. Rizzi 1985, The status of subject clitics in Romance, in Studies in Romance Linguistics, a cura di O. Yaeggli e S. Silva-Corvalán, Dordrecht, Foris, pp. 391-419. $391-419$.

L. Vanelli 1986, Strutture tematiche in italiano antico, in Tema/Rema in italiano a cura di H. Stammerjohan, Tübingen, Narr, pp. 249-263.

L. Vanelli 1987, I pronomi soggetto nei dialetti italiani settentrionali dal Medioevo a oggi, in "Medioevo romanzo", 12/7, pp. 173-211.

L. Vanelli, L. Renzi, P. Benincà 1985, Typologie des pronoms sujets dans les langues in Actes du 17ème Congrès International de Linguistique et Philologie romanes, vol. 3: Linguistique descriptive, Université de Provence-Marseille, Laffitte, pp. 161-176 (anche in it. in Quaderni patavini di linguistica, 5, 1985-86, pp. 49-66. 
Povzetek

\section{K ZGODOVINI ZGRADBE STAVKA V ITALIJANŠČINI: FLORENTINŚČINA V 16. STOLETJU}

Kar zadeva besedni red $v$ stavku in status osebnih zaimkov ( $z$ vrednostjo osebka), je razvojni lok florentinščine natanko tako kot $\mathrm{v}$ francoščini.

V 13. in 14. stol. najdemo nekaj florentinskih literarnih del, $v$ katerih je raba nenaglašenega obglagolskega zaimka sicer poljubna v glavnih stavkih, je pa skorajda obvezna v odvisnikih, torej Dico ch'io vengo 'pravim, da bom prišel', ne pa *Dico che vengo.

Ob koncu 18. stol. je florentinščina prešla iz te faze v drugo: osebni zaimek kot naslonka postaja obvezen. Ta faza je dokumentirana s primeri iz Zannonijevih komedij, torej (Io) i'um m'intendo, (tue) tu un t'intendi, 'na to se ne razumem', itd. Tej analizi pa sledi $v$ članku analiza zaobešenih nenaglašenih zaimkov $\mathrm{v}$ današnji florentinščini.

Še zmeraj slabo poznana je vmesna doba, ta pa je dokaj dobro predstavljena v komedijah G.B. Gellija (1498-1563). Nenaglašeni osebni zaimek je kot osebek ob glagolu obvezen samo pri strukturi stavka osebek-povedek-predmet. Na nekatera odstopanja se v članku posebej opozarja. Za osvetlitev se navaja tole mesto iz Gellija (La sporta, 1550, I, 1):

- Io non credo che $e^{\prime}$ fosse mai la più astuta vecchia di questa. Così foss'ella impiccata, come ella s'è avveduta de' denari che io ho trovati! E per questo - m'è entrato in sospetto, che io non andrei fora e non fare cosa che stessi bene, se io non tornassi a vedere se $e$ ' sono dove io gli nascosi.

'ne verjamem, da bi sploh kdaj bila bolj zvita starka od tele. Da bi jo obesili, ker je opazila denarje, ki sem jih našel! In tako se mi je zbudil sum, da bi moral iti ven in da ne bi bilo dobro, če ne bi pogledal, ali so denarji še tam, kjer sem jih bil skril'.

Če torej sodobna italijanక̌čina ni jezik z ne-nepomembnim obglagolskim osebkom, je to zato, ker je $v$ Cinkvečentu prepustila florentinščino in njene osebne zaimke njihovi lastni usodi. 